\title{
Mark the methods in human embryo research
}

\begin{abstract}
Research on human embryos depends on precious samples that are inherently variable. Rigorous methods reporting should be the goal of published papers in the field.
\end{abstract}

Recent methodological developments have renewed the ethical and policy debates surrounding research on human embryos. Reports of prolonged embryo culture have led some to propose that technical advances are set to collide with the 14-day rule for in vitro culture of these samples. Separately, CRISPR gene editing of early human embryos has raised the deep ethical question of whether, and when, it is acceptable to modify the human germline. These are important discussions and will doubtless continue. Here we raise a more pragmatic point that is nevertheless still a part of ethical practice.

Studies of early human development, critical for understanding this fundamental process in our species, come with a need for scrupulous methods reporting. Although important for all research, methods reporting for work on human embryos-precious by their very nature and by virtue of the difficulty in obtaining them-should ideally be of the highest standard. But scientists working with human embryos are operating in a less than ideal world. They often have little control over many aspects of the embryos they receive. Most are surplus from in vitro fertilization (IVF) clinics and are bedeviled by inherent biological variability.

Unlike the laboratory mouse, humans are genetically outbred; thus human embryos available for research have varied genetic backgrounds. Human embryos also vary in developmental competence, reflected in high miscarriage rates even in natural human reproduction. This is compounded in IVF by the fact that some egg donors are not naturally fertile-because of age or other reasons-and because eggs are obtained after ovarian hyperstimulation. All together, the resulting embryos are heterogeneous in stage and developmental competence, and many may be chromosomally abnormal. The highest-'quality' embryos are used for reproductive purposes, leaving embryos of lower quality available for donation to research.

To add to this biological variability, the embryos a researcher has to work with may be fresh or frozen; the latter will have been prepared by one of multiple different methods, and may have been frozen recently or many years earlier. They could be suspended in one of various media and may have been subjected to additional treatments. Ideally, these details of sample preparation would be available to researchers, but they are sometimes not. Embryos can also be thawed in more than one way.
Variations in in vitro conditions, in genetics, and in embryo quality will affect molecular profiles and developmental outcomes-for instance, the medium an IVF embryo is cultured in has been reported to affect embryo growth rate and birth weight. This is not surprising considering the symphony of critical biological activity that is pre-implantation development. Research results could well be influenced by such variation, a situation that is further confounded by the small numbers of embryos typically available for a given project.

Although some aspects are clearly not in researchers' control, details of sample preparation and embryo quality should be reported in publications to the extent possible and investigated when unknown. Consensus grading schemes for determining embryo quality, for instance, are being developed within the assisted reproductive technology community; these are based mainly on morphological criteria and are used to identify the highestquality embryos for reproductive purposes. A related system could be valuable for embryos used in more basic research.

Some journals, including Nature journals, recommend that researchers report at least the top tier of the BRISQ (Biospecimen Reporting for Improved Study Quality) guidelines for studies using human tissue. Broadly speaking, these guidelines cover both fundamental aspects of the sample (tissue type, anatomical location, clinical status of the donor) and details of how it was collected and processed. Although BRISQ does not specifically deal with human embryos, the guidelines could be extended or used as a template to do so.

For methods development using human embryos, researchers and editors should consider reporting negative results. Were certain hypothetical conditions to be inimical to gene editing human embryos, for example, it would behoove scientists to inform others of their findings even if they find ways to subsequently circumvent these problems. At a minimum, detailed protocols should be provided. It is likely that such practices would also improve record keeping and experimental design.

The collective nature of science becomes even more pressing for work on precious samples-because they are difficult to obtain, politically or ethically sensitive, or both as in the case of human embryos. Especially in such work, we must learn from each other's experiences; this is possible only if they are laid out in detail, methods foremost. 